Please do not remove this page

RMIT

UNIVERSITY

\title{
Effect of polydispersity on the crystallization kinetics of suspensions of colloidal hard spheres when approaching the glass transition
}

Schoepe, Hans-Jaochim; Bryant, Gary; Van Megen, William

https://researchrepository.rmit.edu.au/esploro/outputs/9921860330001341/filesAndLinks?institution=61RMIT_INST\&index=null

Schoepe, H.-J., Bryant, G., \& Van Megen, W. (2007). Effect of polydispersity on the crystallization kinetics of suspensions of colloidal hard spheres when approaching the glass transition. Journal of Chemical Physics, 127(8), 084505-084505. https://doi.org/10.1063/1.2760207

Published Version: https://doi.org/10.1063/1.2760207

Repository homepage: https://researchrepository.rmit.edu.au

(C) 2007 American Institute of Physics

Downloaded On 2023/04/27 00:30:06 +1000 


\title{
Effect of polydispersity on the crystallization kinetics of suspensions of colloidal hard spheres when approaching the glass transition
}

\author{
Hans Joachim Schöpe \\ Institut für Physik, Johannes Gutenberg-Universität Mainz, Staudingerweg 7, D-55128 Mainz, Germany \\ Gary Bryant and William van Megen \\ Department of Applied Physics, Royal Melbourne Institute of Technology, Melbourne, Vic, 3001, Australia
}

(Received 4 May 2007; accepted 26 June 2007; published online 23 August 2007)

\begin{abstract}
We present a comprehensive study of the solidification scenario in suspensions of colloidal hard spheres for three polydispersities between $4.8 \%$ and $5.8 \%$, over a range of volume fractions from near freezing to near the glass transition. From these results, we identify four stages in the crystallization process: (i) an induction stage where large numbers of precursor structures are observed, (ii) a conversion stage as precursors are converted to close packed structures, (iii) a nucleation stage, and (iv) a ripening stage. It is found that the behavior is qualitatively different for volume fractions below or above the melting volume fraction. The main effect of increasing polydispersity is to increase the duration of the induction stage, due to the requirement for local fractionation of particles of larger or smaller than average size. Near the glass transition, the nucleation process is entirely frustrated, and the sample is locked into a compressed crystal precursor structure. Interestingly, neither polydispersity nor volume fraction significantly influences the precursor stage, suggesting that the crystal precursors are present in all solidifying samples. We speculate that these precursors are related to the dynamical heterogeneities observed in a number of dynamical studies. (C) 2007 American Institute of Physics. [DOI: 10.1063/1.2760207]
\end{abstract}

\section{INTRODUCTION}

Hard-sphere-like colloidal suspensions have become an important model system for the experimental study of crystallization and the glass transition for over two decades. ${ }^{1}$ The advantage of these systems over atomic and molecular systems is that the time scales of both the dynamics and kinetics of crystallization are much more accessible, with crystallization occurring over periods from minutes to days. The relative tardiness of the crystallization in these systems is due to the Brownian nature of the motions of the particles and their large size (hundreds of nanometers).

However, crystallization kinetics are also strongly influenced by the particle size polydispersity, a property inherent to all suspensions of colloidal particles. Bolhuis and Kofke ${ }^{2}$ used Monte Carlo techniques to determine the crystal-fluid phase boundaries for polydisperse systems. This work demonstrated that fractionation of particles during crystallization is possible, and in later work they showed that such fractionation increases with volume fraction. ${ }^{3}$ In the phase diagram produced, the polydispersity in the crystal never exceeds a value of $5.7 \%$, indicating the presence of an upper limit to the polydispersity tolerance in a single hard-sphere crystal. Bartlett $^{4}$ suggested that crystallization occurs within multiple fractions of the overall distribution, and then drew attention ${ }^{5}$ to the differences between the quenched and annealed states, and suggested that fractionated crystals are the equilibrium annealed state in a polydisperse hard-sphere system. Other workers, using a range of theoretical techniques, have confirmed the importance of segregation/fractionation. ${ }^{6-10}$ Local size segregation was also predicted for polydisperse particles near a wall. ${ }^{11}$ Finally, taking a different tack, Auer and Frenkel $^{12}$ found, using classical nucleation theory, that the presence of polydispersity leads to an increase in surface free energy and hence a suppression of nucleation.

However, despite the extensive theoretical work, it is only relatively recently that systematic experimental studies of the effects of polydispersity have been carried out. Early works ${ }^{13,14}$ found that increased polydispersity leads to a significant slowing in crystallization kinetics, without significantly affecting particle dynamics. More recent results by Francis et al., using an improved spectrometer, ${ }^{15}$ have furthered studies of the influence of polydispersity in several respects: first, it was established that a particle size distribution with a significant skew to small sizes can lead to unregistered planes of crystallites (a smecticlike phase) $;{ }^{16}$ second, it was shown that symmetrical and highly skewed distributions display qualitatively different growth behaviors, ${ }^{17}$ which suggested a proposed growth mechanism whereby crystallization occurs in conjunction with a local fractionation process near the crystal-fluid interface. By adding a second component, ${ }^{18}$ we were able to demonstrate that this effect could be reproduced, and it was observed that a particle size distribution skewed to smaller sized particles has a stronger effect on the crystal structure than a skew to larger sized particles. For a negative skew, it was found that initial crystallite growth occurred at only a small number of sites, with further crystallization sites developing at later times.

Recently, ${ }^{19}$ we demonstrated that crystallization in colloidal hard spheres occurs in two stages-precursor and induction stages, characterized by the growth of clusters with long lived nonequilibrium structures, followed by a delayed 
nucleation process. We suggested that polydispersity limited growth is responsible for the induction stage and leads directly to the delay in nucleation. Finally, by carefully fractionating a single particle preparation ${ }^{20}$ and characterizing the subtle differences in particle size distribution, we were able to show that very small changes in the polydispersity (from $4.8 \%$ to $5.8 \%$ ) dramatically alter crystallization kinetics $^{21}$ and showed that the length of the induction stage and the delay time prior to nucleation are directly related to the amount of polydispersity.

In this paper, we extend these most recent results by presenting the crystallization kinetics for particles with three different, carefully characterized, polydispersities, as a function of volume fraction from freezing up to the glass transition. From these, we determine the crystallinity, the crystal volume fraction, the crystal size, the absolute number of crystals, and a number of characteristic times, and we investigate the trends in these parameters as functions of polydispersity.

\section{EXPERIMENT}

The experimental methods and analysis used here are described elsewhere ${ }^{17,20}$ and will be summarized here only briefly. The particles consist of a copolymer core of methylmethacrylate and trifluoroethylacrylate, with a stabilizing coating of poly-12-hydroxystearic acid, about $10 \mathrm{~nm}$ thick, bonded to the surface. To reduce the suspension's turbidity, the particles and the suspending solvent, cis-decalin, have refractive indices that are closely matched. Phase diagrams and direct measurements of the force between the stabilizing layers $^{22}$ both show that these particles behave as near perfect hard spheres.

Full details of the fractionation and characterization process are published elsewhere. ${ }^{20}$ Using centrifugation techniques, the stock suspension (XL65-A) was fractionated into two steps, yielding two new samples XL65-B and XL65-C with different particle size distributions (PSDs). The PSDs were characterized using scanning electron microscopy and light scattering. ${ }^{23}$ This yielded polydispersities of $5.8 \%$, $5.3 \%$, and $4.8 \%$, respectively, for XL65-A, XL65-B, and XL65-C. The PSDs are shown in Fig. 1. The first two have distributions slightly skewed to smaller sizes, and the last one has an almost symmetrical, Gaussian distribution. The hydrodynamic radius for all three is $R_{H}=320 \pm 10 \mathrm{~nm}$, which leads to a Brownian time $t_{B}=R^{2} / D_{0}=0.5 \mathrm{~s}$, where $D_{0}$ is the free particle diffusion coefficient.

The equilibrium phase diagram of the least polydisperse sample, XL65-C, was determined, as described previously. ${ }^{24}$ The effective hard-sphere volume fraction was determined by referencing the observed freezing volume fraction to the known freezing volume fraction of hard spheres with a $5 \%$ polydispersity, ${ }^{2,3}$ giving a freezing volume fraction $\phi_{f}$ of 0.505 and a melting volume fraction $\phi_{m}$ of about 0.538 . The scaling factor derived in this way was used for all three polydispersities. This is in contrast to most previous studies, which have referenced the freezing volume fraction to that for the one component hard-sphere system $\left(\phi_{f}=0.494\right)$. A summary of the samples studied is given in Table I.

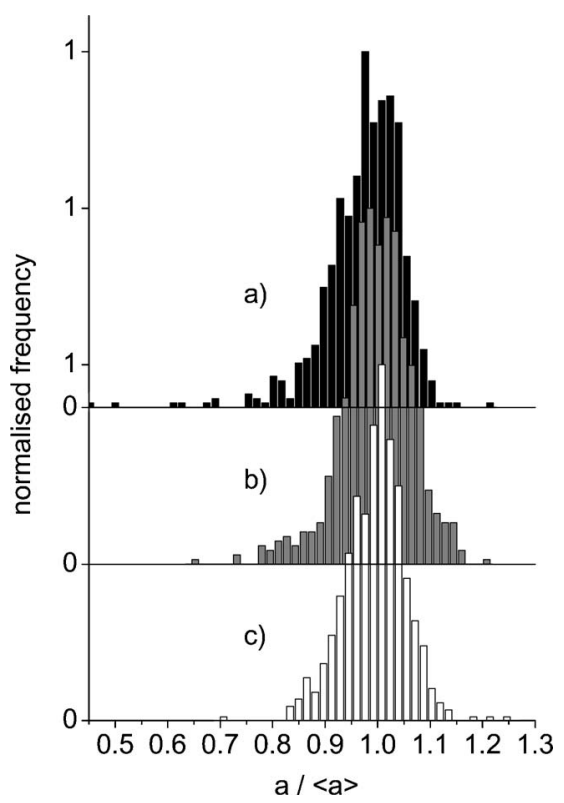

FIG. 1. Particle size distributions of the three polydispersities used. (a) XL65-A, $\sigma=5.8 \%$, skewed; (b) XL65-B, $\sigma=5.3 \%$, skewed; (c) X165-C, $\sigma$ $=4.8 \%$, symmetric. The polydispersity was determined by a combination of EM, SLS, and DLS.

Crystallization kinetics were measured using time resolved laser light Bragg scattering, using a spectrometer that collects the light scattered into the whole Debye-Scherrer cone, which allows statistically meaningful data to be collected at earlier times than previously. ${ }^{15}$ The measured structure factor $S(q, t)$ is used to characterize crystal growth as a

TABLE I. All samples studied in this work, the results of which are presented in Figs. 5-8. Figures 2-4 show examples. Note that measurements on each sample were conducted three to five times, and average results are presented throughout.

\begin{tabular}{|c|c|c|c|}
\hline Latex & Polydispersity (\%) & Volume fraction & Sample designation \\
\hline \multirow[t]{7}{*}{ XL65-A } & 5.8 & 0.514 & $0.514-\mathrm{A}$ \\
\hline & & 0.527 & $0.527-\mathrm{A}$ \\
\hline & & 0.538 & $0.538-\mathrm{A}$ \\
\hline & & 0.542 & $0.542-\mathrm{A}$ \\
\hline & & 0.550 & $0.550-\mathrm{A}$ \\
\hline & & 0.556 & $0.556-\mathrm{A}$ \\
\hline & & 0.57 & $0.57-\mathrm{A}$ \\
\hline \multirow[t]{8}{*}{ XL65-B } & 5.3 & 0.514 & $0.514-\mathrm{B}$ \\
\hline & & 0.524 & $0.524-B$ \\
\hline & & 0.527 & $0.527-\mathrm{B}$ \\
\hline & & 0.538 & $0.538-\mathrm{B}$ \\
\hline & & 0.54 & $0.54-\mathrm{B}$ \\
\hline & & 0.545 & $0.545-\mathrm{B}$ \\
\hline & & 0.556 & $0.556-\mathrm{B}$ \\
\hline & & 0.57 & $0.57-\mathrm{B}$ \\
\hline \multirow[t]{7}{*}{ XL65-C } & 4.8 & 0.516 & $0.516-\mathrm{C}$ \\
\hline & & 0.527 & $0.527-\mathrm{C}$ \\
\hline & & 0.532 & $0.532-\mathrm{C}$ \\
\hline & & 0.538 & $0.538-\mathrm{C}$ \\
\hline & & 0.547 & $0.547-\mathrm{C}$ \\
\hline & & 0.556 & $0.556-\mathrm{C}$ \\
\hline & & 0.57 & $0.57-\mathrm{C}$ \\
\hline
\end{tabular}




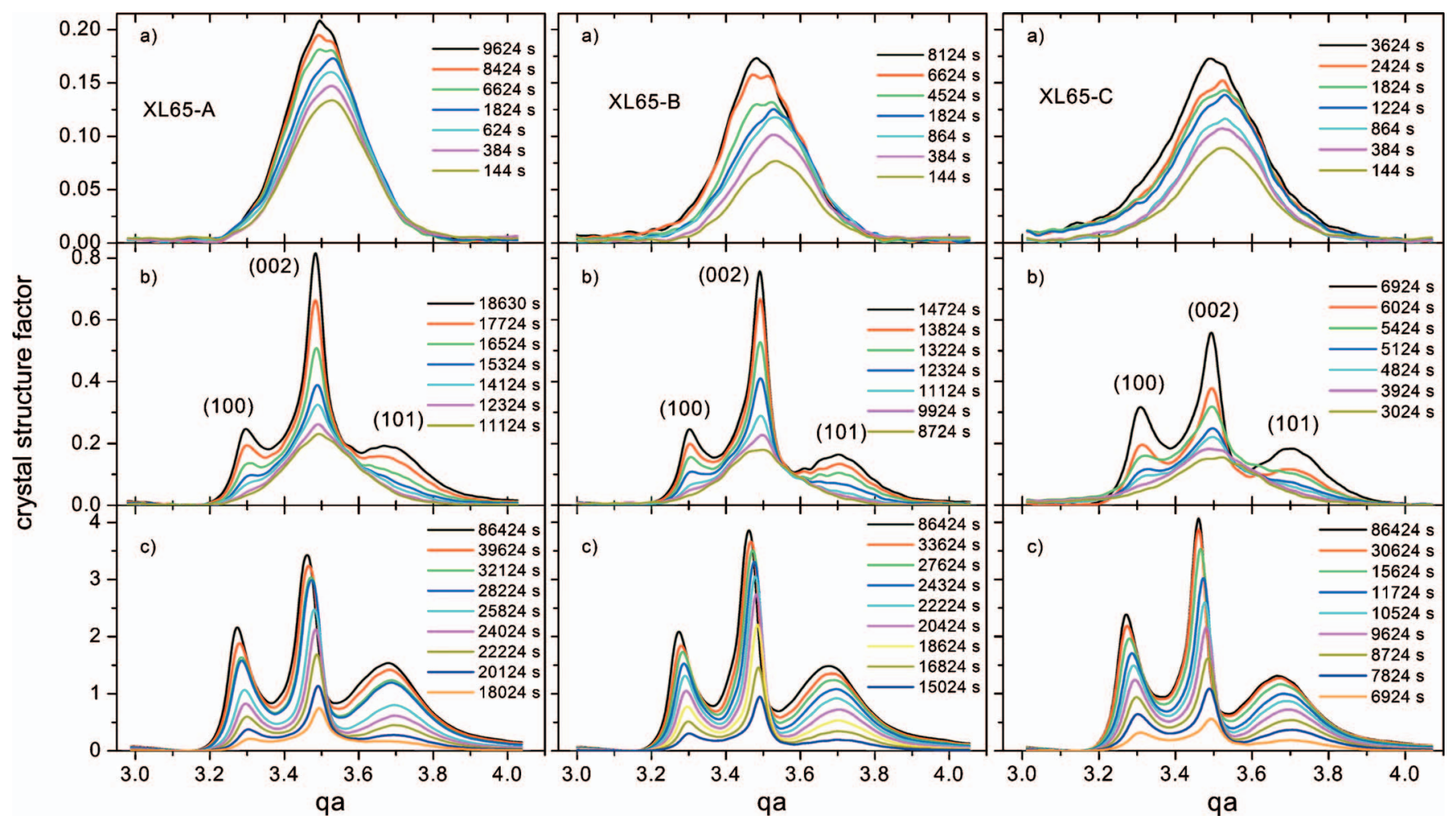

FIG. 2. (Color) Time evolution of the crystal structure factor at $\phi=0.538$, around the melting volume fraction, for the three different polydispersities, divided into three different time periods. (a) shows the precursor stage with only one broad peak evident; (b) shows the conversion stage, where the other peaks begin to appear, but overall growth is slow; and (c) shows the final nucleation phase, where all three peaks grow rapidly. Note the different vertical scales for each phase.

function of the scattering vector $q$ and elapsed time $t$. The crystalline structure factor $S_{\text {xtal }}$ is extracted from the measured data by subtracting the fluid background $\beta(t) S_{\text {fluid }}(q)$, as described by Harland and van Megen, ${ }^{25}$

$$
S_{\text {xtal }}(q, t)=S(q, t)-\beta(t) S_{\text {fluid }}(q),
$$

where the fluid structure factor is obtained $60 \mathrm{~s}$ after stopping the shear melting process. The parameters extracted from the crystalline structure factor are as follows:

(1) The crystallinity $X(t)$ (the fraction of the sample which is crystalline) is determined by integrating the structure factor over the area of the main Bragg reflection,

$X(t)=c \int S_{\text {xtal }}(q, t) d q$,

where $c$ is obtained after normalizing to the value obtained from the equilibrium phase diagram.

(2) The crystalline volume fraction, which is determined from the peak maximum $q_{\max }(t)$ estimated from Gaussian fits to the peak of the structure factor,

$\phi_{\text {xtal }}(t)=\frac{2\left[q_{\max }(t) R\right]^{3}}{9 \sqrt{3} \pi^{2}}$.

(3) The average linear dimension of the crystals, $\langle L(t)\rangle$, which is determined from $\delta q(t)$, the full width at half maximum of the peak,

$$
\langle L(t)\rangle=\frac{2 \pi K}{\delta q(t)},
$$

where $K=1.0747$ is the Scherrer constant for a spherical shaped crystal. ${ }^{26}$ From these basic parameters, the following quantities can be determined:

(4) The number of crystals in the scattering volume,

$N_{\text {xtal }}(t)=\frac{X(t) V_{\text {scatt }}}{\left\langle L^{3}(t)\right\rangle}=\frac{X(t) V_{\text {scatt }}}{\alpha\langle L(t)\rangle^{3}}$,

where $V_{\text {scatt }}\left(\sim 360 \mathrm{~mm}^{3}\right)$ is the volume illuminated by the laser, and $\alpha \approx 1.25$ (Ref. 27) connects the average crystal size cubed with the average crystal volume (see Ref. 17 for discussion).

\section{RESULTS AND DISCUSSION}

Figure 2 shows the time evolution of the crystalline structure factor, broken into three time intervals, for each of the three polydispersities at a volume fraction near melting $(\phi=0.538)$. The top panels show the early times following the quench, designated the induction stage, where precursor structures lead to one broad peak growing slowly in intensity while the width remains nearly constant. The middle panels show the intermediate time period, where two other peaks start to appear. The three peaks can now be identified as the hexagonal close packed (hcp) (100), (002), and (101) reflections. The main reflection is also the face centered cubic (fcc) (111) reflection—colloidal hard spheres crystallize as a 

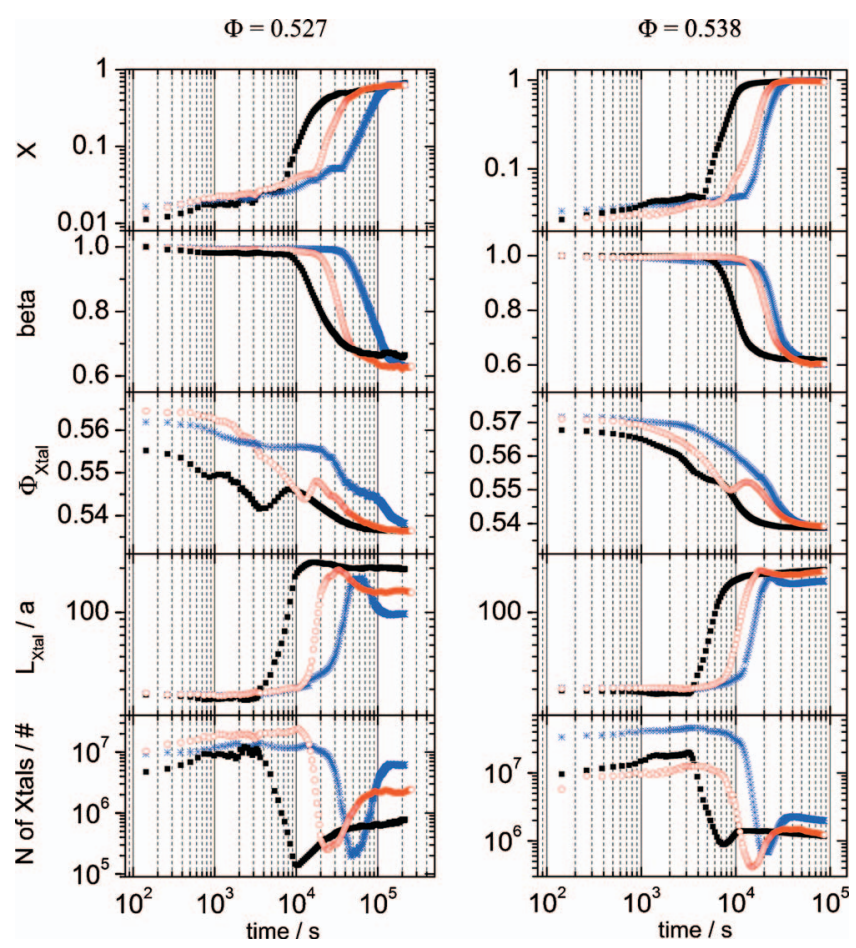

FIG. 3. (Color) From top to bottom: crystallinity, factor $\beta$ scaling the fluid background, crystalline volume fraction, average linear dimension, and absolute number of crystals for volume fractions in the coexistence region: $\phi=0.527$ and 0.538 . Shown for the different polydispersities: (blue crosses) XL65-A, (red circles) XL65-B, and (black squares) XL65-C.

mixture of fcc and hcp structures designated random hexagonal close packed (rhcp). ${ }^{28}$ This stage is designated the conversion stage, where the width of the central peak decreases significantly while the crystallinity starts to rise more rapidly, and the crystallites evolve from the initial precursor structures to a rhcp structure. The bottom panels show the final or nucleation stage, where the structure does not change significantly but the intensity increases rapidly. Note that the nucleation observed here is due to the homogeneous nucleationthere was no evidence of heterogeneous nucleation, which can be readily distinguished both by eye and spectroscopically. ${ }^{16,29}$

Figures 3 and 4 show the time dependent behavior for four of the eight volume fractions studied. Figure 3 shows volume fractions in coexistence $(0.527)$ and at around melting $\left(0.538=\phi_{m}\right)$, and Fig. 4 shows volume fractions above melting (0.556) and near the glass transition (0.57). Several parameters extracted from the crystalline structure factors are shown: the crystallinity $X$ [Eq. (2)], the factor $\beta$ scaling the fluid background [Eq. (1)], the crystalline volume fraction $\phi_{\text {xtal }}\left[\right.$ Eq. (3)], the average crystal size $L_{\text {xtal }}[$ Eq. (4)], and the number of crystals $N$ [Eq. (5)].

The three time intervals shown in Fig. 2 can be seen most clearly for samples in the coexistence region shown in Fig. 3. The induction stage comprises approximately the first 1.5 decades of the time window. During this time, both $X$ and $N$ increase weakly, while the average crystallite size $L$ remains essentially constant at around $L \sim 30$ radii.

The subsequent sharp increase in $X$ can be broken down into two stages: the first, referred to above as conversion, shows $L$ increasing by about one order of magnitude, while
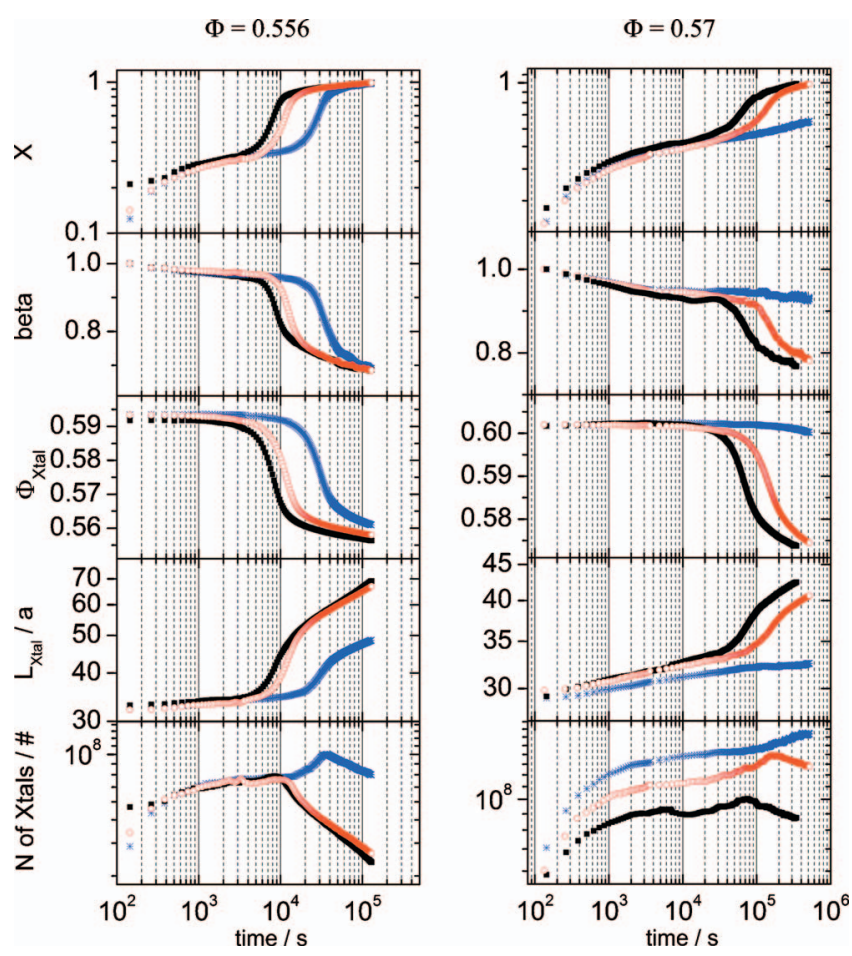

FIG. 4. (Color) Same as for Fig. 3 but for volume fractions beyond melting: $\phi=0.556$ and 0.57 .

$N$ decreases by up to two orders of magnitude. In this conversion stage, the average volume of the crystallites increases faster than the total crystal volume in the sample. This, combined with the appearance of the 100 and 101 peaks (Fig. 2), indicates that some precursors are converting into rhcp crystallites, with a large number of precursors disappearing: either the crystallites are growing by the attachment and registration of precursors or some of the precursors are melting as others grow.

During the second stage of the increase in $X, L$ is seen to reach a maximum and then decrease for all samples except XL65-C at $\phi=0.538$, which increases slowly. At this stage, $X$ and $N$ exhibit their most pronounced rate of increase. Accordingly, this stage is referred to as nucleation. For all samples, toward the end of the time window, $N$ decreases slowly, while $L$ increases slowly, caused by crystal ripening.

Figure 3 shows that the samples with different polydispersities have similar behavior in the coexistence region. The most significant effect of polydispersity in this region is to extend the induction stage - by almost an order of magnitude in going from $\mathrm{C}$ to $\mathrm{A}$ (a change in polydispersity of around $1 \%)$.

The most interesting aspect of these data is that in the induction stage, the average linear dimension of the precursors $(L \sim 30$ radii) shows no significant variation with either volume fraction or polydispersity. The clear implication is that these are the entities that seed the conversion to the crystalline phase.

Note that the volume fraction of the precursors (at $t \sim 100 \mathrm{~s})$ is significantly above the average volume fraction of the suspension. The determination of the volume fraction using Eq. (3) presumes that structures are rhcp. However, it is clear from the discussion above that this is not the case. 
Given that in this stage the crystalline structure factor has only one peak in our spatial window, the state of organization in the induction stage can, at most, consist of smecticlike unregistered layers of particles, as discussed previously. ${ }^{16}$

Registration of these layers, indicated by the development of multiple reflections, is delayed by fractionation-the expulsion from the precursors of particles whose radii lie in the wings of the PSD. Obviously, the broader the PSD, the more fractionation is required before registration can be effected.

The calculated value of $\phi_{x t a l}(t)$ will depend on the composition and degree of registration, i.e., how close the actual structure is to rhcp, as well as of the average particle size inside the observed crystals. The interplay of these could be responsible for the nonmonotonicity evident in $\phi_{\text {xtal }}(t)$ at low $\phi$.

Moving beyond the coexistence region (Fig. 4), a number of significant qualitative differences become apparent. First, the crystallinity increases more appreciably in the induction stage, so that by the time conversion takes place, approximately $30 \%-40 \%$ of the volume is bound up in precursors-compared to only around 5\% in the coexistence region.

Second, at the higher volume fractions, the drop in the number of crystals dividing the two processes becomes very small or disappears altogether. The transition between the precursor and the crystal nucleation is then simply given by the point inflection of this curve. The important distinction here is that the steepest increase in $X$ and $L$ is now accompanied by an increase in $N$. This suggests, in contrast to the behavior in coexistence, that essentially all precursors are converted into crystals. Further, the intensity of the main nucleation process is reduced; $N$ increases only about $5 \%$ $15 \%$.

Third, when the main nucleation event begins to plateau, only about $80 \%$ of the sample is crystalline, and this is followed by a slow increase in crystallinity. $N$ is observed to rise until the end of the main nucleation event, and then drops continuously, while $L$ rises sharply during the nucleation event, and then continues to rise slowly during the slow final process. In other words, the last stage of the transition is dominated by ripening, which is very pronounced compared to the samples in coexistence.

Interestingly, at this volume fraction, the two least polydisperse samples (XL65-B and XL65-C) are very similar, and there is a much smaller difference in crystallization time than is observed at lower volume fractions. XL65-A, however, is still significantly slower.

For XL65-A, the 100 and 101 reflections are never seen during the course of the measurements at the highest volume fraction - there is no evidence of nucleation or ripening. Instead, the precursor structures grow slowly in both size and number, until they fill the sample volume.

To learn more about the influence of volume fraction and polydispersity on the crystallization process, we present several quantities as functions of volume fraction. Figure 5 shows (a) the maximum observed crystallite size and (b) the crystallite size at the end of crystallization, for the three

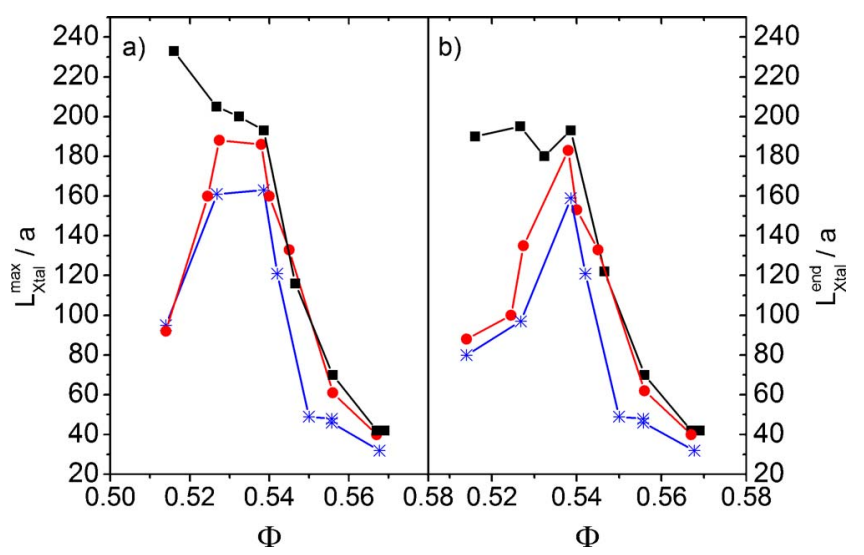

FIG. 5. (Color online) Average crystal size as a function of volume fraction for the three samples: (a) maximum crystal size observed during the crystallization process and (b) final crystal size at the end of crystallization. (Blue crosses) XL65-A, (red circles) XL65-B, and (black squares) XL65-C.

polydispersities as functions of volume fraction. Three observations can be made from these data. First, for the more polydisperse samples (XL65-A, B), both the maximum and final average crystal size shows a distinct maximum at around $\phi_{m}$. Second, this maximum is not seen for the least polydisperse samples (XL65-C) - instead, the maximum crystallite size decreases continuously as a function of volume fraction, with the largest crystals being produced at the lowest volume fractions, as has been observed previously. ${ }^{30}$ Finally, the crystal sizes for all three polydispersities converge at high volume fractions.

To learn more about the induction stage, Fig. 6 shows

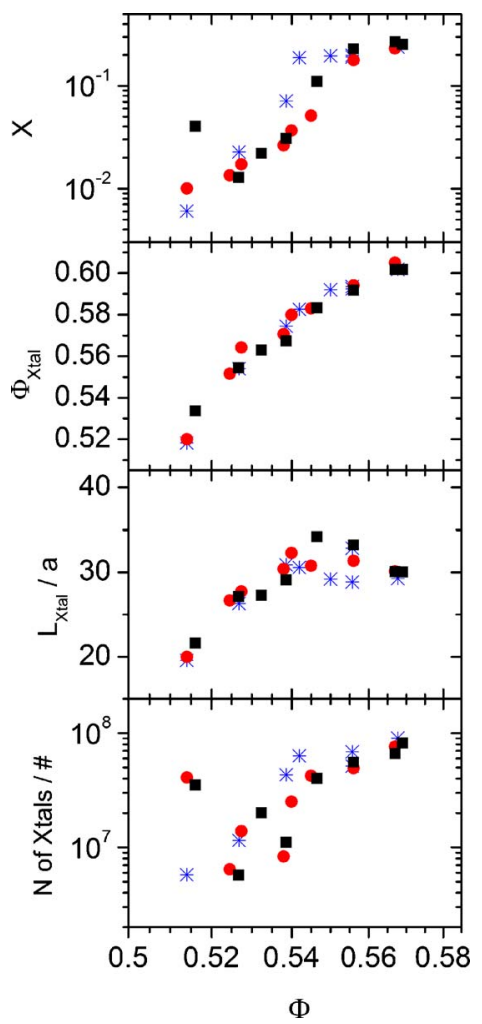

FIG. 6. (Color online) Characteristic properties shown in Figs. 3 and 4, at the start of the precursor stage. Data are averaged in time over the first $600 \mathrm{~s}$. 


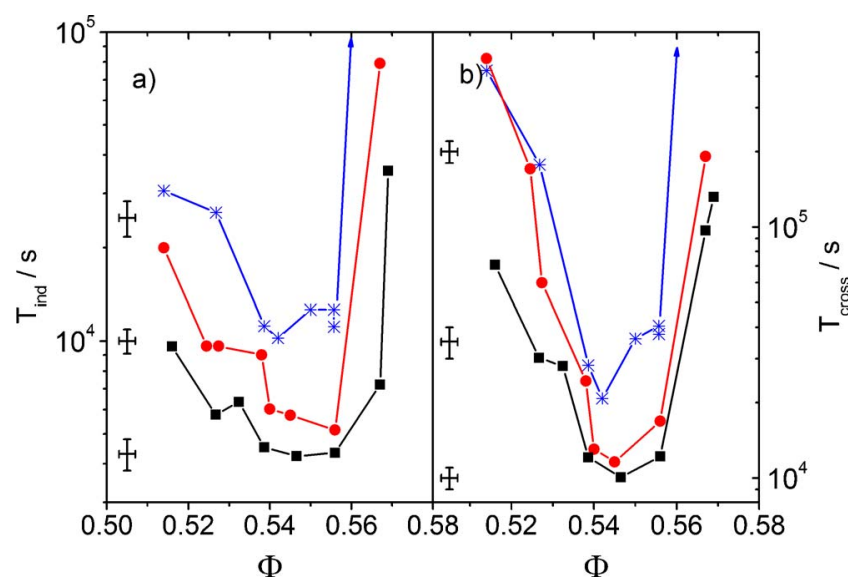

FIG. 7. (Color online) Characteristic times as a function of volume fraction for the three samples: (a) induction time and (b) crossing time. (Blue crosses) XL65-A, (red circles) XL65-B, and (black squares) XL65-C.

the values of $X, \Phi_{\text {xtal }}, L$, and $N$ averaged over the first $600 \mathrm{~s}$, as functions of volume fraction. With the exception of results at the lowest volume fraction, all four parameters increase monotonically with $\phi$, and there is little evidence of any systematic dependence on polydispersity. Note that the lowest volume fraction is very near freezing and has the largest errors. These results indicate that the precursor formation is independent of polydispersity.

Figure 7 shows characteristic times as functions of volume fraction: (a) shows the induction time, i.e., the duration of the induction stage, and (b) shows the crossing time denoting the end of the nucleation process, as defined elsewhere. ${ }^{25}$ The arrows in the XL65-A data set at high volume fractions indicate that the sample at 0.57 does not crystallize.

These characteristic times show a volume fraction dependence, with minima around 0.55 , consistent with a previous work. ${ }^{25}$ More interestingly, however, both the characteristic times are very sensitive to the polydispersity. This is in contrast to the properties which characterize the precursor structures, which expose no such sensitivity.

Figure 8 shows the volume fraction dependence of the apparent "crystalline" volume fraction, at four different times during the crystallization process: (a) the beginning of the induction stage, (b) the beginning of the nucleation stage, (c) where the nucleation rate has its maximum, and (d) the end of the experiment. The solid lines indicate the total volume fraction of the sample. Differences between the polydispersities are not evident at the beginning of the induction stage - the structures that form have a significantly higher density than the overall volume fraction of the sample. It is well known that crystallites are initially compressed relative to the equilibrium volume fraction. ${ }^{25}$ This representation allows us to see how the volume fraction changes with both polydispersity and volume fraction.

The difference between the measured volume fraction and the equilibrium line decreases as crystallization proceeds. Within the errors, by the end of crystallization, the final volume fractions are equal to the melting volume fraction for $\phi \leqslant \phi_{m}$ and equal to the sample volume fraction for $\phi \geqslant \phi_{m}$, as would be expected for a fully crystallized sample.

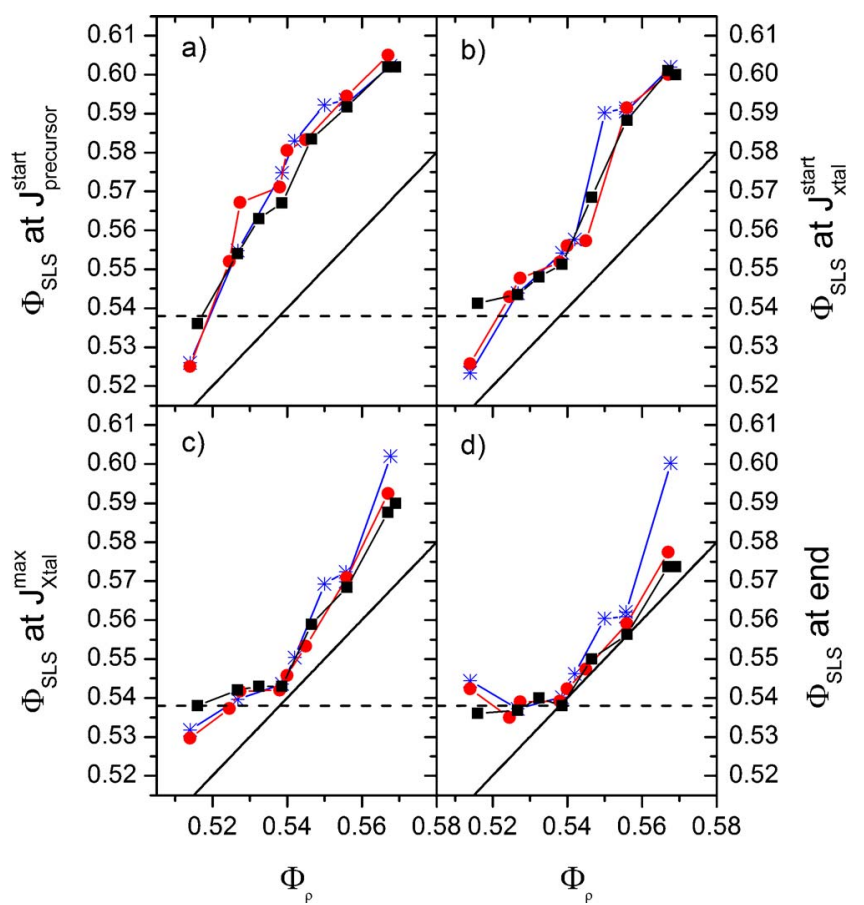

FIG. 8. (Color online) Crystal volume fraction as a function of the sample volume fraction: (a) at the start of the precursor nucleation, (b) at the start of the crystal nucleation, (c) where the crystal nucleation has its maximum, and (d) at the end of the crystallization process. (Blue crosses) XL65-A, (red circles) XL65-B, and (black squares) XL65-C. The solid lines indicate the total volume fraction of the sample. The dashed line represents the melting volume fraction $\phi_{m}$.

At the lowest volume fraction in the precursor stage [Fig. 8(a)], XL65-B and C have a slightly lower than equilibrium volume fraction-this is presumably due to the fact that the precursors are mainly composed of larger than average sized particles, leading to a lower apparent volume fraction. Conversely, at the end of the crystallization process [Fig. 8(d)], these samples are mainly composed of smaller than average sized particles, leading to a higher than equilibrium apparent volume fraction. At $\phi=0.57$, the most polydisperse sample XL65-A remains in the compressed induction stage and never reaches equilibrium.

\section{CONCLUSIONS}

We have presented a comprehensive investigation of the solidification scenario in suspensions of colloidal hard spheres for three different particle size distributions over a range of volume fractions starting close to freezing and ending close to the glass transition. From these results we identify the following:

(i) An induction stage where the structure factor around the main interlayer reflection and the properties derived from it change quite slowly. The size of the precursors stays nearly constant, while their number increases significantly. During the induction stage, only one interlayer reflection is observed [nominally the hcp (002) reflection], implying that the structures consist of unregistered layers of particles.

(ii) A conversion stage identified by a strong increase in the average linear crystal dimension and a weak in- 
crease in crystallinity while the number density of crystallites decreases. This decrease is significantly reduced above melting. The precursor structures are converted to rhcp structures, indicated by the emergence of the 100 and 101 reflections.

(iii) Nucleation, where the number of crystallites and the crystallinity shows its strongest increase, while the behavior of the average crystal size $L$ varies with volume fraction-at the lowest volume fraction, it decreases, while at higher volume fractions, it continues to increase. This stage is suppressed with increasing undercooling (overpacking) above melting.

(iv) A ripening stage where [as in (ii) $] N$ decreases while $L$ increases, but where the amount of crystal remains essentially fixed. This stage is enhanced with increasing undercooling for volume fractions above melting.

The duration of the induction stage increases with polydispersity. The local fractionation required to facilitate conversion from unregistered layers to rhcp crystallites becomes increasingly difficult as either polydispersity or volume fraction is increased. At volume fractions near the glass transition, this process is entirely frustrated, and the sample is locked into a compressed crystal precursor structure. We speculate that these precursors are related to the dynamical heterogeneities observed in a number of dynamical studies. $^{31-37}$

The most important point to emerge from these studies is that in the early stages of solidification, all samples behave in a similar fashion, regardless of volume fraction or polydispersity. This universality suggests that the crystal precursors are present in all solidifying samples and are an important link between crystallization and the glass transition.

\section{ACKNOWLEDGMENT}

One of the authors (H.J.S.) would like to thank the Alexander von Humboldt Foundation for providing financial support.

${ }^{1}$ P. N. Pusey and W. van Megen, Nature (London) 320, 340 (1986); P. N. Pusey, in Liquids, Freezing and The Glass Transition, Les Houches Session 51, 1989, edited by J.-D. Levesque (Les Houches, North-Holland,
Amsterdam, 1991)

${ }^{2}$ P. G. Bolhuis and D. A. Kofke, Phys. Rev. E 54, 634 (1996).

${ }^{3}$ D. A. Kofke and P. G. Bolhuis, Phys. Rev. E 59, 618 (1999).

${ }^{4}$ P. Bartlett, J. Chem. Phys. 109, 10970 (1998).

${ }^{5}$ P. Bartlett, J. Phys.: Condens. Matter 12, A275 (2000).

${ }^{6}$ R. P. Sear, Europhys. Lett. 44, 531 (1998).

${ }^{7}$ R. M. L. Evans and C. B. Holmes, Phys. Rev. E 64, 1404 (2001).

${ }^{8}$ H. Xu and M. Baus, J. Chem. Phys. 118, 5045 (2003).

${ }^{9}$ M. Fasolo and P. Sollich, Phys. Rev. Lett. 91, 068301 (2003).

${ }^{10}$ M. Fasolo and P. Sollich, Phys. Rev. E 70, 041410 (2004).

${ }^{11}$ I. Pagonabarraga, M. E. Cates, and G. J. Ackland, Phys. Rev. Lett. 84, 911 (2000).

${ }^{12}$ S. Auer and D. Frenkel, Nature (London) 413, 711 (2001).

${ }^{13}$ S. I. Henderson, T. C. Mortensen, S. M. Underwood, and W. van Megen, Physica A 233, 102 (1996).

${ }^{14}$ S. I. Henderson and W. van Megen, Phys. Rev. Lett. 80, 877 (1998).

${ }^{15}$ P. Francis, S. Martin, G. Bryant, W. van Megen, and P. A. Wilksch, Rev. Sci. Instrum. 73, 3872 (2002).

${ }^{16}$ S. Martin, G. Bryant, and W. van Megen, Phys. Rev. Lett. 90, 255702 (2003).

${ }^{17}$ S. Martin, G. Bryant, and W. van Megen, Phys. Rev. E 67, 061405 (2003).

${ }^{18}$ S. Martin, G. Bryant, and W. van Megen,Phys. Rev. E 71, 021404 (2005).

${ }^{19}$ H. J. Schöpe, G. Bryant, and W. van Megen, Phys. Rev. Lett. 96, 175701 (2006).

${ }^{20}$ H. J. Schöpe, O. Marnette, and G. Bryant (to be published).

${ }^{21}$ H. J. Schöpe, G. Bryant, and W. van Megen, Phys. Rev. E 74, 060401(R) (2006).

${ }^{22}$ G. Bryant, S. R. Williams, I. K. Snook, Q. LinMao, E. Perez, and F. Pincet, Phys. Rev. E 66, 060501(R) (2002).

${ }^{23}$ G. Bryant, S. Martin, A. Budi, and W. van Megen, Langmuir 19, 616 (2003).

${ }^{24}$ S. E. Paulin and B. J. Ackerson, Phys. Rev. Lett. 64, 2663 (1990).

${ }^{25}$ J. L. Harland and W. van Megen, Phys. Rev. E 55, 3054 (1997).

${ }^{26}$ A. L. Patterson, Phys. Rev. 56, 978 (1939).

${ }^{27}$ K. M. Dobrich, C. Rau, and C. E. I. Krill, Metall. Mater. Trans. A 35A, 1953 (2004)

${ }^{28}$ P. N. Pusey, W. van Megen, P. Bartlett, B. J. Ackerson, J. G. Rarity, and S. M. Underwood, Phys. Rev. Lett. 63, 2753 (1989).

${ }^{29}$ A. Heymann, A. Stipp, C. Sinn, and T. Palberg,J. Colloid Interface Sci. 207, 119 (1998).

${ }^{30}$ W. van Megen and S. M. Underwood, Nature (London) 362, 616 (1993).

${ }^{31}$ M. M. Hurley and P. Harrowell, Phys. Rev. E 52, 1694 (1995).

${ }^{32}$ E. R. Weeks, J. C. Crocker, A. C. Levitt, A. Schofield, and D. A. Weitz, Science 287, 627 (2000).

${ }^{33}$ W. Kob, J. Phys.: Condens. Matter 11, R85 (1999).

${ }^{34}$ S. C. Glotzer, J. Non-Cryst. Solids 274, $342(2000)$

${ }^{35}$ W. K. Kegel and A. v. Blaaderen, Science 287, 290 (2000)

${ }^{36}$ R. Richert, J. Phys.: Condens. Matter 14, R703 (2002).

${ }^{37}$ J. C. Conrad, P. P. Dhillon, E. R. Weeks, D. R. Reichman, and D. A. Weitz, Phys. Rev. Lett. 97, 265701 (2006). 